\title{
Une année riche d'événements illustrant le dynamisme de la Société internationale francophone d'éducation médicale (SIFEM)
}

Après notre congrès de Bruxelles, qui a réuni près de 300 personnes, parmi lesquelles de nombreux enseignants paramédicaux, et qui fut un franc succès, aux dires des participants et selon l'analyse des évaluations réalisées, notre société a continué à développer ses axes stratégiques en formation médicale pour le bien de tous, enseignants et étudiants du monde des sciences de la santé. En voici quelques exemples.

\section{Le congrès SIFEM 2015 et ses suites}

Il est encore possible d'avoir accès aux enregistrements des sessions et conférences plénières sur le site de la faculté de l'Université catholique de Louvain. Il suffit de se rendre à l'adresse http:// podcast.mede.ucl.ac.be/ puis, après avoir accepté les conditions légales et générales, de choisir le chapitre PODICONF dans la barre de menu et de sélectionner le dossier SIFEM 2015 dans la liste affichée à gauche de l'écran. Les différentes conférences plénières sont alors accessibles ; elles peuvent être enregistrées sous format $\mathrm{mp} 4$.

Un des deux thèmes clefs du congrès était « $\mathrm{La}$ responsabilité sociale des formateurs en sciences de la santé ». La richesse des discussions et des recherches exécutées dans le domaine a permis une synthèse publiée dans un numéro thématique de la revue Pédagogie Médicale ${ }^{[1-6]}$. Le projet, soutenu à la fois par notre société et par la Conférence internationale des doyens et des facultés de médecine d'expression française (CIDMEF), a continué son essor. Un comité de pilotage a été créé et une phase de recherche-action a été lancée, à laquelle contribuent 58 facultés de 18 pays différents. Ce projet de recherche action vient tout récemment d'être distingué en recevant le Prix international AFMCCharles Boelen pour la responsabilité sociale 2016, décerné par l'Association des facultés de médecine canadiennes. Il faut en féliciter chaleureusement le comité de pilotage.

Le deuxième thème du congrès concernait la problématique de l'approche pédagogique par compétences. Il a fait l'objet d'exposés présentant à la fois des cadres conceptuels et plusieurs expériences développées dans des cadres institutionnels ou disciplinaires. Les différentes contributions seront également publiées dans un numéro thématique de la revue, en cours de préparation.

\section{Les axes de développement et d'appui aux enseignants en sciences de la santé}

\section{Approbation éthique des projets de recherche en éducation en sciences de la santé}

La possibilité de pouvoir solliciter l'approbation éthique par un comité habilité d'un projet recherche 
en éducation pour la santé comportant des interventions qui concernent des étudiants ou des patients, a été exprimée à plusieurs reprises. Il s'agit notamment de pouvoir répondre aux exigences des comités de rédaction des revues dans la perspective d'une publication. Les comités d'éthiques ou de protection des personnes habilités en recherche biomédicale ou clinique se déclarent en effet souvent incompétents dans le domaine de la recherche en éducation. Pour répondre aux besoins de la communauté francophone, la SIFEM va mettre en place un tel comité, constitué de quatre ou cinq personnes mandatées par le conseil d'administration de la société. Les membres intéressés, soit pour participer à ce comité, soit pour soumettre un projet, sont invités à prendre contact avec le secrétariat de la SIFEM, qui leur fera parvenir les modalités pratiques.

Le projet de formation internationale francophone en pédagogie des sciences de la santé (F2IFS2)

De moins en moins de personnes contestent le besoin d'une formation aux bases pédagogiques pour tout (futur) enseignant dans le domaine des sciences de la santé. De multiples formations existent, que ce soit sous forme de diplôme universitaires (DU) ou interuniversitaires (DIU) ou de masters. La plupart d'entre elles exigent la présence des étudiants à des ateliers ou séminaires et, à notre connaissance, peu de formation sont disponibles à distance. La SIFEM, en partenariat avec le conseil pédagogique de la CID$\mathrm{MEF}$, a jugé utile de proposer une formation internationale francophone, basée à la fois sur une formation à distance (sous forme de Massive Open Online Course -MOOC) et sur un parcours mixte, incluant la présence à un nombre limités d'ateliers (d'où des coûts plus réduits), un travail personnel dans son lieu d'origine et un suivi à distance par des tuteurs compétents. Une formation plus complète pouvant aller jusqu'au master de 60 (un an) ou de 120 crédits (deux ans) est également envisagée. La particularité de ce projet est en effet de proposer une valorisation et une capitalisation des activités de formation sous forme de crédits ECTS (European credit transfer system), tels qu'ils ont été mis en place dans le cadre de la réforme dite «Bologne » ou Licence-Master-Doctorat (LMD). Sous réserve d'homologation par les instances universitaires, ce système permet de valoriser dans le cursus des formations ou des participations à des congrès, et/ou à des recherches et des activités d'enseignement (pour autant qu'elles soient évaluées). La formation comporterait donc plusieurs niveaux qui prennent en compte et valorisent les activités réalisées dans les niveaux inférieurs.

Les objectifs de cette formation progressive peuvent se résumer comme suit : acquisition de connaissances et de compétences pédagogiques dans le cadre de programmes proposés en distanciel et en présentiel, selon quatre niveaux :

- Niveau 1 : Massive Open Online Course (MOOC) : module de 20 heures, permettant d'acquérir un niveau de connaissances pédagogiques de base, sur inscription mais ouvert à tous et gratuit.

- Niveau 2 : Small Prived Online Course (SPOC) : module de 120 heures en distanciel et présentiel, avec approfondissement des connaissances acquises par le MOOC. Les activités en présentiel seront notamment dédiées à la formation sur des thèmes comportant une dimension «logisitique », tels que les examens cliniques objectifs structurés (ECOS) ou encore les dispositifs recourant à la simulation.

- Niveau 3 : diplomation (niveau diplôme ou certificat d'université) après obtention des niveaux 1 et 2 et soutenance d'un mémoire.

- Niveau 4 : délivrance de masters 1 et 2 en pédagogie des sciences de la santé, sur la base de 120 heures de formation délivrée en distanciel et présentiel.

Si vous souhaitez vous impliquer dans certains projets cités ci-dessus, n'hésitez surtout pas à nous contacter.

Jean-François Denef Secrétaire général de la SIFEM

Dominique Maillard Présidente de la SIFEM

Mailto : jfdenef@gmail.com 


\section{Références}

1. Pestiaux D. La responsabilité sociale des institutions de formations en santé. Pédagogie Médicale 2015; 16:163-5.

2. Lefève C, Gateau V. Responsabilité sociale : un point de vue philosophique. Pédagogie Médicale 2015;16: $167-70$

3. Boelen C. Responsabilité sociale des institutions de formation : où en sommes-nous ? Pédagogie Médicale $2015 ; 16: 171-4$

4. Grand'Maison P, Ladner J, Maherzi A, Poitevien G, Poitras J, Duplain R et al. Facultés de médecine francophones et responsabilité sociale : approche stratégique 2015-2020. Pédagogie Médicale 2015; 16:175-82

5. Moineau G. Responsabilité sociale des facultés de médecine et accréditation. Pédagogie Médicale 2015;16:183-8

6. Ladner J, Maherzi A, Poitevien G, Pestiaux, Grand'Maison P, Gomes J, et al. Responsabilité sociale des facultés de médecine francophones: organisation, résultats et leçons apprises du projet de recherche-action international des facultés de médecine francophones. Pédagogie Médicale 2015;16: 189-200. 